RUNNING HEAD: A CONCERT FOR BABIES

To appear in Developmental Science

\title{
A concert for babies: Attentional, affective, and motor responses in an infant audience
} Haley E. Kragness ${ }^{1}$, Bryna Berezowska², \& Laura K. Cirelli ${ }^{1}$

1Department of Psychology, University of Toronto Scarborough, Scarborough, Ontario, Canada ${ }^{2}$ CultureLink, Department of Settlement \& Community Services, Toronto, Ontario, Canada

\section{Corresponding author: Laura K. Cirelli, laura.cirelli@utoronto.ca}

Data availability: The data and analysis code can be accessed at https://osf.io/4hvdw/

Funding statement: This research was supported by an Insight Grant (\# 435-2019-0202) from the Social Sciences and Humanities Research Council (SSHRC) to LKC.

Conflict of interest: The authors have no conflicts of interest to report.

Ethics approval statement: The research was approved by the University of Toronto Research Ethics Board and was conducted in compliance with recognized standards for experimentation with human subjects.

Acknowledgments: We thank The Music Box team: Bryna Berezowska, Chris Thornborrow, Sonja Rainey, Alex Berezowsky, Mary-Dora Bloch-Hansen, Germaine Liu, and Danika Lorèn. We thank the Four Seasons Performing Arts Centre and Canadian Opera Company for performance space. Thank you to Aghilan Aiyadurai, Vishnija Asokan, Saghar Baqizada, Deepika Elango, Irene Guerroro, Shenwei Hu, Sherry Huang, Ella Lawrence, Kevin Naismith, Laiba Rizwan, Julia Santiago, Sangavi Sivananthan, Chella Velkannen, and Idila Yogeswaran for assistance with data collection and annotation. 


\section{Research Highlights}

2 - Infants' responses to live musical performances are shaped by the music, by their

$3 \quad$ caregivers, and by their own musical histories

4 - During a concert for babies, a playsong more effectively elicited infant attention and

5 smiles than a lullaby, especially when caregivers were interactive

- Infant attention was more coordinated with their own caregiver than with other caregivers watching the same show 


\section{Abstract}

2 Many of our most powerful musical experiences are shared with others, and researchers have

3 increasingly investigated responses to music in group contexts. Though musical performances

4 for infants are growing in popularity, most research on infants' responses to live music has

5 focused on solitary caregiver-infant pairs. Here, we report infants' attentional, affective, and

6 motor responses to live music as audience members. Two groups of caregiver-infant (6-18

7 months) pairs (50 total) watched a short musical performance with two song styles - lullaby and

8 playsong. Caregivers were instructed to watch passively or interactively. The playsong captured

9 more infant attention and, especially in the interactive condition, elicited more infant smiles.

10 Notably, infant attention was more coordinated with their own caregiver than a random

11 caregiver, and infants with no experience attending group musical events in the past were

12 especially attentive to the performance. Infants were more likely to generate movements when

13 parents remained still. Overall, infants' responses to live musical performance in an audience

14 were influenced by song style, caregiver behavior, and their own musical histories.

15 Keywords: infant development, singing, music, coordinated attention, infant-directed song, 16 dance 


\section{Introduction}

Across our lives and throughout human history, music is frequently experienced in social settings. Caregivers gently rock and sing to their newborn infants, teens share playlists with their closest friends, and older adults remember favorite songs even after losing other important memories. These social and emotional experiences may have been crucial for the evolution of musicality in human history (Savage et al., 2020). In contemporary life, the social benefits of sharing music may underlie why we invest substantial money, time, and effort into attending concerts despite availability of high-quality recordings (Brown \& Knox, 2017). Indeed, most young adults report that their most intense musical experiences occurred with other people, almost always at a live music event such as a concert or festival (Lamont, 2011).

In recognition of this, recent research in the arts has shifted to measuring audience responses outside of traditional lab environments. While solitary listening contexts can sometimes evoke chills and pleasure similar to listening with others (Egermann et al., 2011; Belfi et al., 2021), merely seeing other audience members elicits more vigorous movement to music (Dotov et al., 2021). Likewise, live performances enjoyed with a group encourage more audience movement, more extreme physiological responses, and more emotional coordination compared to recorded playbacks (Shoda et al., 2016; Swarbrick et al., 2019; Upham \& McAdams, 2018). Taken together, results suggest that both performer interaction and the collective experience shared with fellow audience members contribute to the power of live performances.

Our earliest musical experiences tend to occur in a more constrained social context the caregiver-infant dyad. Caregivers sing softly to their infants to lull them to sleep, and in lively ways to play or distract them from distress (Cirelli \& Trehub, 2020; Trehub \& Gudmundsdottir, 2015). These two song styles have distinct impacts on the behavior and physiological state of the dyad (Cirelli, et al., 2020; Rock et al., 1999). The soft, slow, low singing associated with 
1 lullabies relaxes the singer and the infant listener alike. Playsongs, on the other hand, are sung

2 faster, louder, and higher, and are often accompanied by rhythmic movements, capturing

3 attention and stabilizing arousal levels in both members of the dyad (Cirelli et al., 2020). Live

4 caregiver song is a frequent element of infant interactions across the globe, despite the

5 prevalence of recorded music heard via electronic toys and television (Ilari, 2005; Mendoza \&

6 Fausey, 2021).

$7 \quad$ Infants' experiences with music and the performing arts may be embedded in the wider

8 community, as well. Caregivers often bring their infants and young children to musical programs

9 hosted in the community. Globally, professional shows geared toward toddler and infant

10 audiences have risen steeply over the last few decades, though the extent to which

11 performances incorporate music, dance, theatrical performance, and audience interaction is

12 highly variable (Drury \& Fletcher-Watson, 2017; Fletcher-Watson et al., 2014). Often, such

13 performances take place in novel community environments outside of the infants' home, such

14 as a library, park, or auditorium. The singer and musical material are likely unfamiliar, and there

15 are other families in attendance. Does music manipulate infants' attention and behavior in such

16 stimulating contexts? Do caregivers play a role in modulating their infants' experience? Despite

17 the rise in popularity of these performance events, there have been few attempts to characterize

18 infants' experiences of these events (but see Barbosa, et al., 2021).

19 In the present experiment, we investigated how song style, caregiver behavior, and

20 infants' past musical experiences influenced their engagement as audience members during a

21 live musical performance ( $\sim 30$ caregiver-infant pairs at each of two performances, $\sim 60$ total

22 pairs). The performance included excerpts from an infant-directed opera called The Music Box,

23 a collaborative composition led by the second author. The show includes vocals and

24 instrumental accompaniment. Using video data, we examined how infants' attention, affect, and

25 rhythmic movements were influenced by song type (lullaby, playsong) and caregiver

26 engagement (passive, interactive). Given previous laboratory work, we predicted that the 
1 playsong would capture infant attention more effectively than the lullaby, and explored whether

2 song type would also influence infant rhythmic movements and affect. Interactivity between

3 caregivers and infants could also play an important role in directing and engaging infants'

4 responses. However, how interactivity might alter infants' behavior across dependent variables

5 is not clear. For instance, interactivity might either facilitate infants' attention to the show, or

6 alternatively could represent a distraction. Here, we explored the effect of caregiver

7 engagement, but did not have directional hypotheses. Finally, we explored the dyadic nature of

8 this experience by examining coordinated attention between caregiver and infant pairs.

Method

\section{Participants}

Data were collected across two performances in April 2019, attended by 61 families with infants. The present sample size is nearly twice that of previous laboratory studies of similar

14 effects (Cirelli et al., 2020), and maximized the number of infants that could be in attendance

15 simultaneously due to space constraints and availability. Three families did not provide their

16 infants' age and three additional infants were outside of the target age range (6 to 18 months).

17 Four further babies were not visible on any camera (three at the first and one at the second

18 performance) and one infant was fussy. These 11 infants were excluded, leaving a total of 50

19 infants in our analyses (22 at Show 1 and 28 at Show 2; $M$ age $=348.2$ days/11.45 months, SD 20 age $=90.77$ days $/ 2.98$ months; 19 girls and 31 boys) .

21 According to caregiver report, no infants in the sample had any suspected

22 developmental disabilities. On average, our sample was economically wealthier than the 23 average family in Toronto (median 2019 household income $\sim 71,000$ CAD): 42\% of the families

24 in our sample reported household income over $\$ 120,000 /$ year, $44 \%$ of families reported income 25 between $\$ 60,000$ and $\$ 120,000$ year, and $10 \%$ of families reported income less than $26 \$ 60,000 /$ year (2 families did not respond to this question). Out of 45 families who provided 
1 language background information, half of the families indicated that their infant was exposed

2 exclusively to English in home/daycare settings, while the other half indicated their infant was

3 also exposed to one or more other languages at least $10 \%$ of the time, including French (10),

4 Cantonese (4), Persian/Farsi (4), Arabic (2), Mandarin (2), Gujurati (1), Katchi (1), Korean, (1),

5 Kurdish (1), Polish (1), Shona (1), Spanish (1), and Tamil (1). Twenty-two infants had a

6 caregiver with at least 5 years of music training (instrument or voice), of which 10 had

7 caregivers who still played their instrument (or voice) regularly. Twenty-three families said their

8 baby had previously attended some form of baby music class.

9 Infants were recruited from the University of Toronto Scarborough Developmental

10 Database and through targeted social media advertisements. All procedures were approved by

11 the University of Toronto Research Ethics Board.

Procedure

The musical performance included excerpts from an opera specifically for babies called

15 The Music Box, composed collaboratively by the second author and other professional

16 musicians. Two professional opera singers, a pianist, and an auxiliary percussionist performed.

17 Families either attended a morning performance (Show 1) or an afternoon performance (Show

18 2). The show opened with an instrumental overture (not analyzed). The performance then

19 included a playsong ( 2m16s in length) followed by a lullaby ( 2m54s in length). After a $\sim 5-$

20 minute break, the performance was repeated, with the song order for lullaby and playsong

21 reversed. Both songs borrowed musical elements from well-known children's songs ("The Itsy

22 Bitsy Spider" and Brahms' "Lullaby and Goodnight" for the playsong and lullaby, respectively)

23 but included novel lyrics, melodies and rhythmic patterns. The songs were performed in an

24 operatic and infant-directed singing style. Both songs were in a major key and spanned a similar

25 vocal pitch range. The playsong was, on average, faster than the lullaby, and contained more 
rhythmic and tempo variability (see details in Supplemental Table 1). Each session lasted about 20 minutes.

Caregivers completed consent, a demographics questionnaire, and the Music@Home

4 questionnaire (Politimou et al., 2018) before arriving. This is a valid and reliable tool used to

5 calculate a general score reflecting an infant's home musical environment, with subscales

6 capturing caregiver beliefs about music and development, caregiver initiation of singing and

7 music making, and the child's active engagement with music (for the distribution of infants'

8 scores on the Music@Home scales, see Supplemental Figure 1). Infants sat on their caregiver's

9 lap in chairs facing the performance, arranged in 3 long rows (see Figure 1). Once families were

10 settled, they were welcomed by the experimenter. The experimenter explained to caregivers

11 that they would watch a show comprising three short musical pieces, followed by a very brief break, and then the show would repeat. The caregivers in Show 1 were asked to simply hold

13 their infants without moving or singing along (passive condition) before the break. After the

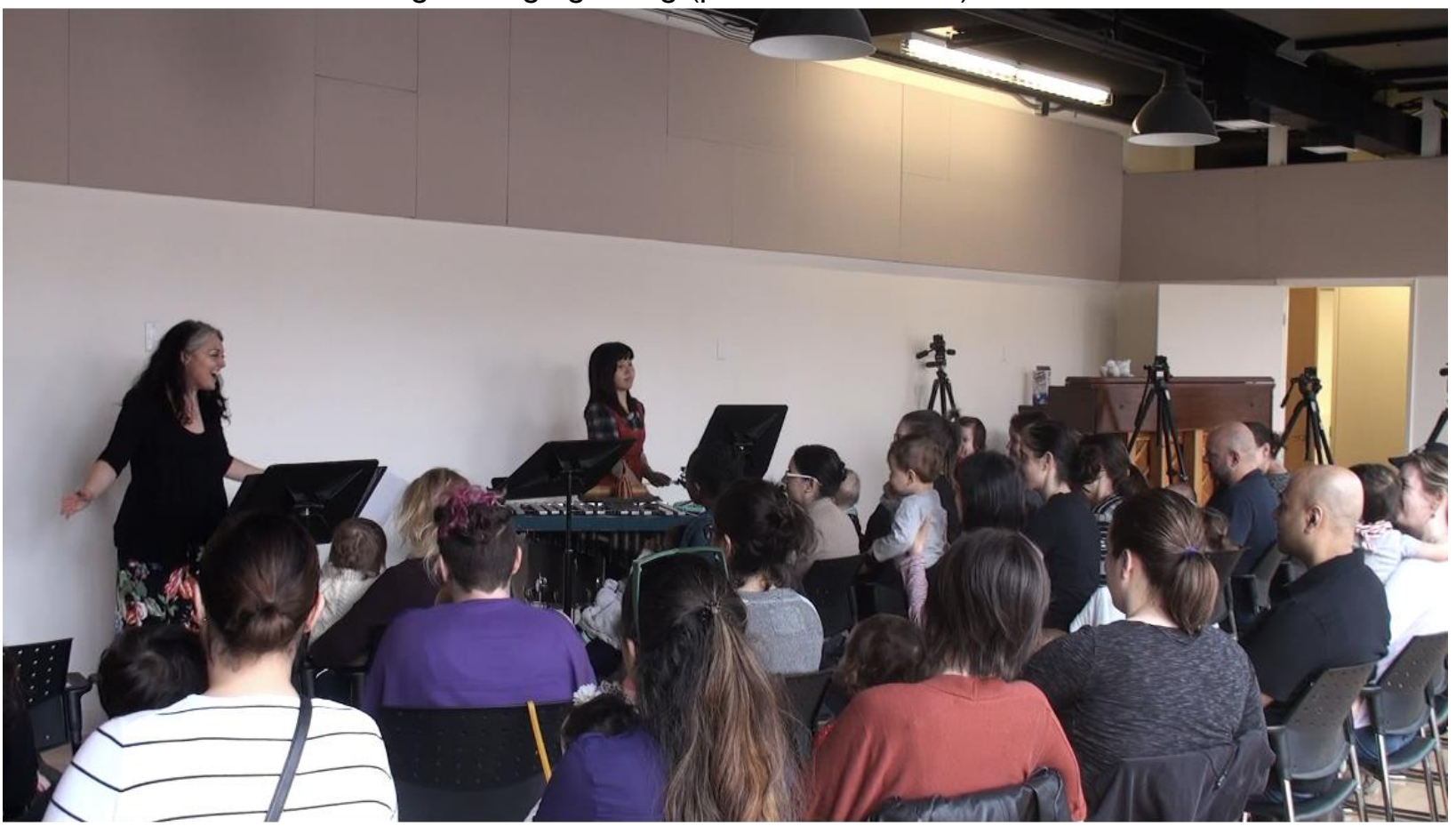

Figure 1. The performance took place in an auxiliary room of the city's performing arts center. The performers (singers, pianist, and auxiliary percussionist) were positioned at the front of the room. The audience was in three rows with an aisle in the middle for easy access. Cameras on tripods were positioned around the audience to capture the infants' behaviors. 
break they were asked to provide their infants with an interactive musical experience by gently

2 moving them to the beat of the music (interactive condition). The caregivers in Show 2 were

3 instructed to be interactive in the first half and to remain passive in the second half.

$5 \quad$ Video Recording, Processing, and Coding

To capture infant responses to the performance, 8 cameras (Akaso EK7000) were

7 placed around the audience on tripods. A cropped video was then created for each of the four

8 trials (lullaby-passive, lullaby-interactive, playsong-passive, playsong-interactive) for each

9 participant using DaVinci Resolve (Blackmagic Design). Trained research assistants, naïve to

10 condition and hypotheses, annotated infant behaviors from videos played without sound. Details

11 about each dependent measure can be found in Supplemental Table 2. Inter-coder agreement

12 over $75 \%$ was considered acceptable for inclusion (Portney \& Watkins, 2009). Percent

13 agreement for epoch-based measures ranged between $89 \%$ and $97 \%$. Agreement for duration-

14 based measures ranged from .82 to .93 (Pearson's $r$ ). See Supplemental Materials for

15 additional details about co-coder agreement. The primary coder's annotations were used for all

16 co-coded analyses, except for rhythmic movement (see below).

\section{Infant Attention}

Attention was coded using ELAN Linguistic Annotator (https://tla.mpi.nl/tools/tla-

20 tools/elan/, Lausberg \& Sloetjes, 2009). Videos were first divided into $2 \mathrm{~s}$ epochs using

21 Segmentation Mode. In each epoch, coders indicated if the infant glanced 1) in the general

22 direction of the performance and/or 2) to their own caregiver (1-yes, 0-no: no minimum duration

23 for a glance). Infant attention was discernible for $98 \%$ of epochs. Note that within a single $2 \mathrm{~s}$

24 epoch, infants could receive a 1 for both of these measures. 

4 for $99 \%$ of epochs.

5

\section{Caregiver Attention}

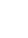

\section{Infant Affect}

\section{Analyses}

Caregiver attention was coded using the same $2 \mathrm{~s}$ epochs created to code infant attention, and the same coding scheme described above. Caregiver attention was discernible

A coder identified the onset time and offset time of positive facial expressions (smiling, crying) and negative facial expressions (frowning, crying). Emotion measures were represented as a proportion of time that they were expressing that emotion out of the time their facial expression was available for coding.

\section{Infant and Caregiver Rhythmic Movement}

Coders watched each clip and identified the onset and offset of each burst of rhythmic movement. Rhythmic movements were defined as the repetition of a particular body movement at least twice within a $2 \mathrm{~s}$ window. Coders identified windows of time when the infants and caregivers independently generated movements in a rhythmic way. Trials where infant movements were coded as highly ambiguous ( $2 \%$ of trials) were excluded from further analyses. For co-coded videos, one coder's ratings were randomly selected to be used in the analyses, and rhythmic movement was represented as a proportion of time they were moving rhythmically out of the time their bodies were visible for coding.

We used linear mixed effects models (LMEM; glmmTMB package; Brooks et al., 2017) in $\mathrm{R}$ (version 3.6.3) to evaluate the effects of predictors on infant and caregiver behavior. We included song condition (lullaby, playsong) and caregiver engagement condition (passive, interactive) as fixed effects, and participant as a random effect to account for the repeated 
measures nature of the design. For each analysis, song condition and caregiver engagement

2 condition were contrast-coded (playsong $=1$, lullaby $=-1$; interactive $=1$, passive $=-1$ );

3 therefore, coefficients represent the effect of each factor at the average level of the other (i.e.,

4 main effects). In a final analysis, we examined whether infant age (days), previous experiences

5 with group music events (baby music classes), and infants' scores on the general factor of the

6 Music@Home survey (Politimou et al., 2018) were associated with infant behaviors. Data and

7 analysis scripts are available on Open Science Framework (https://osf.io/4hvdw/).

8

\section{Results}

\section{Infant Attention to the Performance}

Infants spent a substantial proportion of time watching the performance. Forty-five infants $(90 \%)$ watched the performance during more than $50 \%$ of epochs, and $22(44 \%)$ watched the performance in over $75 \%$ of epochs. Given prior lab-based work, we predicted that infants would pay more attention to the performance during the playsong conditions than the lullaby conditions. There were no significant effects or interactions with caregiver engagement ( $p$ 's $>.503)$, but there was an effect of song type $(B=0.095, S E=.011, z=8.291, p<.001)$, indicating that infants paid more attention to the performance during the playsong $(81 \%$ of epochs contained looks) than the lullaby (62\% of epochs contained looks) across both engagement conditions (Figure 2; Supplemental Table 4).

The significant effect of song condition held over both halves of the show, whether the play song was first or second in song order ( $p$ 's <.001) - in fact, the difference between proportions of looking to each song in the first half of the show (lullaby $=60 \%$, playsong $=78 \%$ ) is nearly identical to the difference in looking to each song in the second half of the show (lullaby $=63 \%$, playsong $=83 \%$ ). A direct comparison of the model with vs. without the song position factor indicated that including it did not improve model fit significantly $(p=.157)$. 


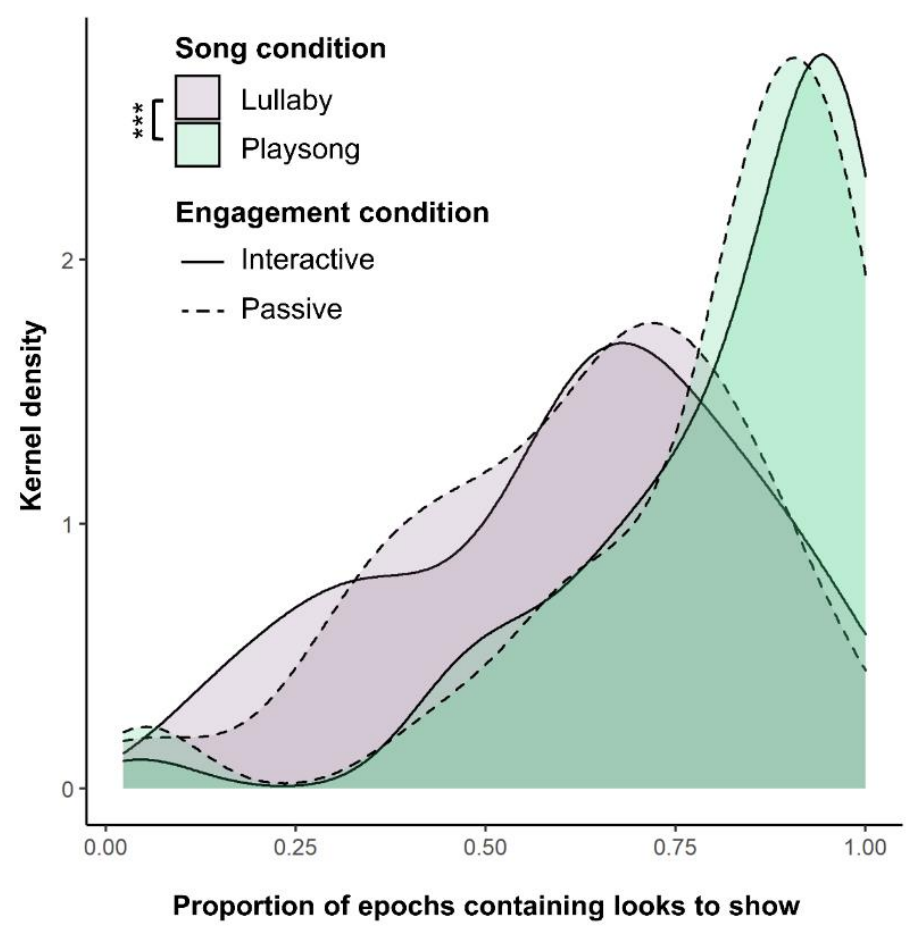

Figure 2. Kernel density plots (smoothed histograms) depicting the proportion of time infants attended to the show during each song and engagement condition. ${ }^{*} p<.05,{ }^{* *} p<.01,{ }^{* *} p<.001$

\section{Coordinated Attention Between Infants and Caregivers}

3 Second, we explored whether infant-caregiver attention was coordinated during the

4 performance. Consistency in infant-caregiver attention was calculated for each trial by

5 calculating the proportion of epochs during which infants and their own caregiver both looked

6 toward the performance, toward each other, or both looked to neither the performance nor each

7 other. As a comparison, we also calculated the consistency between each infant's attention and

8 a randomly-selected caregiver watching the same performance. Coordinated attention was

9 significantly higher for caregiver-infant pairs (57\%) than for randomized pairs $(53 \%), B=.023$,

$10 S E=.010, z=2.340, p=.017$, indicating that coordinated attention between infants and their 
1 own caregivers was greater than chance levels between each infant and a random caregiver

2 watching the same show (Figure 3). We observed the same pattern of results if we considered

3 only looks to the performance or each other (in other words, simultaneous looking towards

4 neither was omitted), $p=.032$.

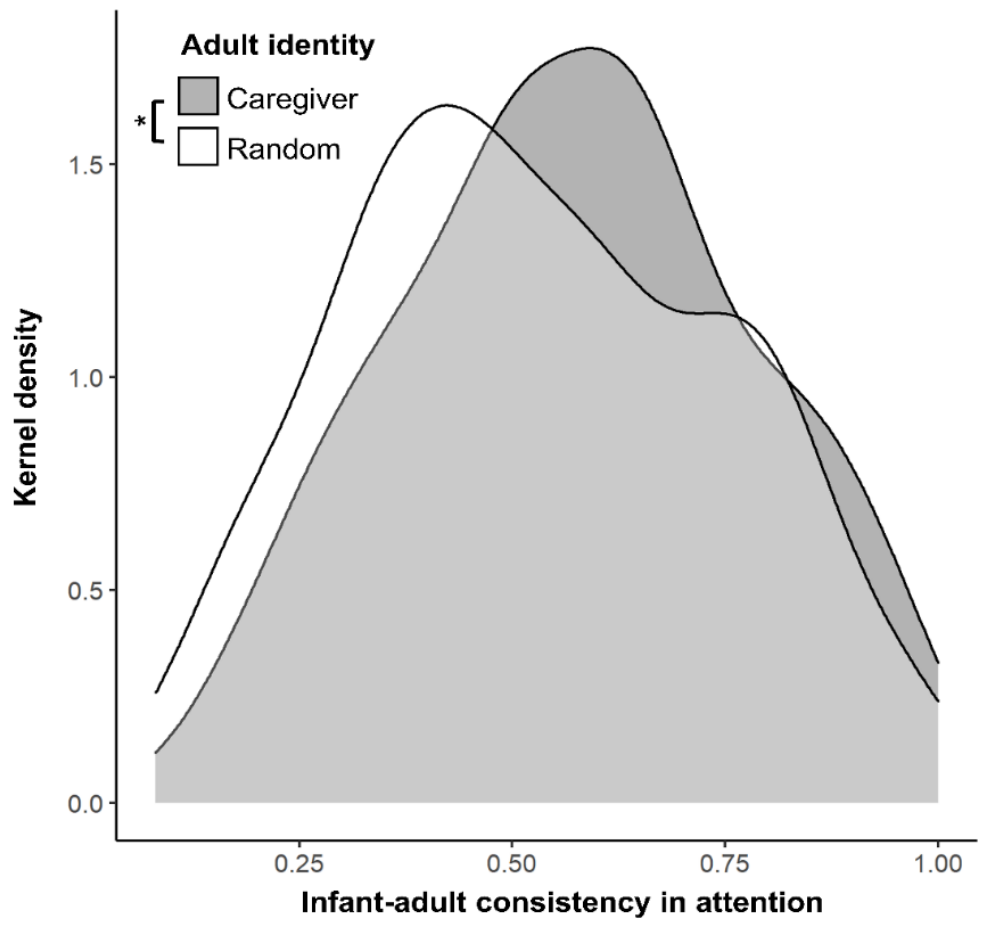

Figure 3. Kernel density plots (smoothed histograms) depicting the proportion of time infants' attention was consistent with either their own caregiver or a random caregiver (both looking at the show, at each other, or at neither).

${ }^{*} p<.05,{ }^{* *} p<.01,{ }^{* \star *} p<.001$

6

7 Infant Affect

There were two time windows in the playsong that encouraged the caregiver to tickle

9 their baby (one starting about 30 seconds in and lasting $\sim 3$ seconds, and a second starting about 56 seconds in and lasting $\sim 20$ seconds). These time windows were omitted to ensure that 
1 differences in affect between song conditions were associated with the song rather than with

2 being tickled.

Overall, infants expressed positive affect $7 \%$ of the time, and 37 out of the 50 infants did

4 so at least once. The proportion of time participants expressed positive affect was compared

5 across song type (lullaby, playsong) and caregiver engagement (passive, interactive)

6 conditions. There was a significant song condition by engagement condition interaction $(B=$

$70.023, S E=0.010, z=2.233, p=.026$; see Supplemental Table 5 and Figure 4A). To interpret

8 the interaction, the same model was evaluated separately for the playsong and lullaby

9 conditions. The effect of engagement condition was only significant during the playsong: infants

10 smiled more during the interactive than passive condition for the playsong $(B=0.036, S E=$

$110.015, z=2.425, p=.015)$, but there was no difference during the lullaby $(B=-0.013, S E=$

$120.012, z=-1.144, p=.253)$. The cameras were positioned to optimize attention coding, so there

13 were some trials in which the infants' face was turned away from the camera (and towards the

14 show), and therefore trials varied substantially in how visible the infants' face was: on average,

15 the infants' facial expression could be judged about $50 \%$ of the trials; in contrast, their attention

16 and movement could be judged for $>98 \%$ of the time. We conducted a multiverse analysis

17 (Steegen et al., 2016) to investigate whether the interaction term was consistently significant

18 when retaining only trials with a certain threshold of visibility, ranging from $1 \%$ to $100 \%$. The

19 effect was significant for $53 \%$ of the possible comparisons, indicating uncertainty about the

20 robustness of this effect; therefore, this result should be interpreted cautiously pending

21 additional confirmatory data (see Supplemental Material for additional details). Expressions of

22 negative affect were extremely rare (identified on average $1 \%$ of the time, and expressed only

23 by 6 out of the 50 infants), but notably were nearly only observed in the lullaby condition (see

24 Figure 4B). 


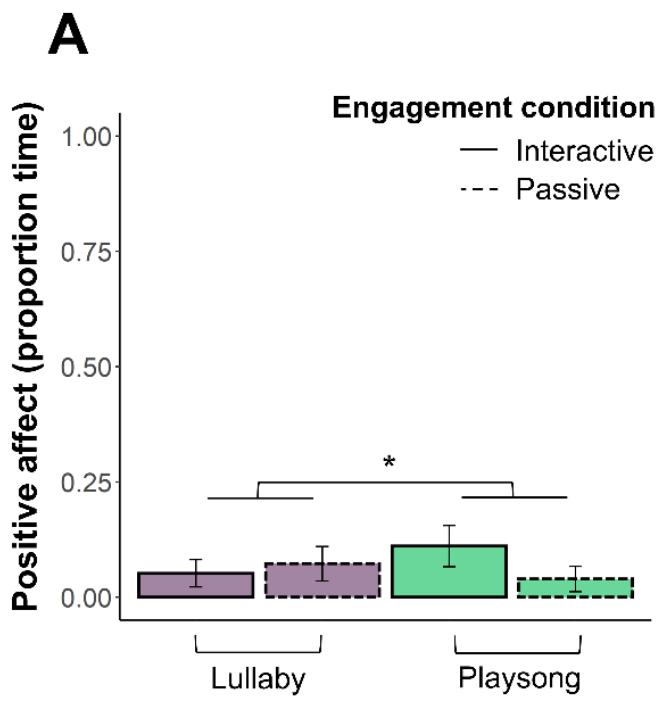

\section{B}

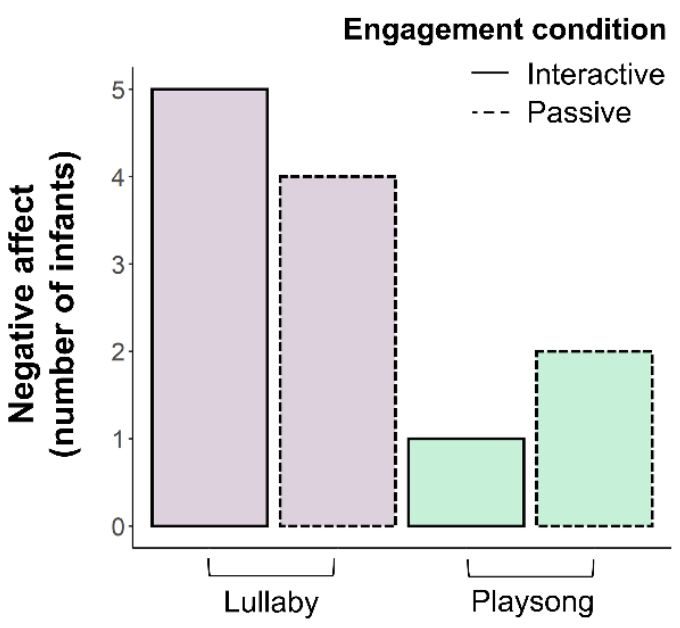

Figure 4. (A) Depicts the proportion of time infants showed positive facial expressions (smiling, laughing) during each trial. Error bars represent $95 \%$ withinsubjects confidence intervals. (B) Depicts the number of infants who expressed negative affect (frowning, crying) during the trial.

${ }^{*} p<.05,{ }^{* *} p<.01,{ }^{* *} p<.001$

Infant Rhythmic Movements

Infant-generated rhythmic movement occurred on $41 \%$ of trials, and $82 \%$ of infants moved rhythmically at least once. Although many infants moved at some point, movements

5 were generally brief - the mean time spent moving across all trials was only $4 \%$. Common

6 movements included clapping, swaying, and leg bouncing. There was a significant effect of

7 engagement condition, such that infants spent more time moving rhythmically during the

8 passive $(M=5 \%)$ compared to the interactive $(M=3 \%)$ condition, $B=-0.011, S E=.005, z=-$

$92.175, p=.030$ (see Supplemental Table 6). In other words, infants appeared to move

10 rhythmically more during the trials when their caregivers were not moving them. 

$7<.001$.

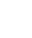

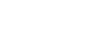

\section{Caregiver Behavior}

To determine whether caregivers followed the experimenter instructions, we compared caregivers' behavior on the interactive vs. passive trials. Three caregiver-infant pairs were present for only half of their respective shows (either passive or interactive, but not both), and were thus excluded for this analysis. A paired $t$-test indicated that caregivers moved rhythmically more often during the interactive condition (52\%) than passive condition $(7 \%), t(46)=11.706, p$

We also examined whether caregivers were more attentive to their babies during the interactive than passive condition. A greater proportion of epochs contained caregiver attention directed towards their infant in the interactive condition (63\%) than the passive condition (46\%), $t(46)=5.802, p<.001$

\section{Influences of Age, Music@Home, and Previous Musical Experiences}

Finally, we conducted post-hoc analyses to investigate whether infants' age or previous musical experiences were related to each of the coded behaviors (Supplemental Table 7).

Collapsing across trials, we conducted simple correlations between each dependent variable, their age, and their scores on the full scale and subscales of the Music@Home questionnaire. Only the Parent Initiation of Music-Making subscale of the Music@Home survey was significantly related to infants' rhythmic movement, $r(48)=.279, p=.049$, but the association would not survive a correction for multiple comparisons and should therefore be treated as highly exploratory.

An unpaired $t$-test did not indicate that the infants who had ever previously attended a music class $(N=23)$ or not $(N=27)$ differed in age, $t(48)=1.066, p=.292$. Whether the infants had attended a previous baby music class did not affect the amount of time they moved rhythmically, $t(48)=0.422, p=.675$, nor the amount of positive affect they displayed, $t(48)=$ $0.258, p=.798$, but it did affect their interest in watching the show, $t(48)=2.726, p=.009$. For 
1 the infants who had never attended a music class, approximately $77 \%$ of visible epochs

2 contained looks to the show, compared to $65 \%$ for infants who had attended at least one music

3 class in the past.

4

5

\section{Discussion}

Attending live musical performances with other audience members often constitutes our most rewarding and enjoyable musical experiences. This has never been more evident than during the COVID-19 pandemic, during which most in-person performances of concerts and performing arts were noticeably absent from our lives. In this study, we investigated infants' behavior while they watched a live music performance in a moderately-sized audience. Overall, most infants appeared to find the performance engaging - on average, their attention was captured by the performance about $70 \%$ of the time. Infants with no experience attending group musical events in the past were especially attentive, perhaps due to the relative novelty of the situation. While overt expressions of positive affect like smiles were fairly infrequent (7\%), expressions of negative affect were extremely rare (1\%). This aligns with findings from Barbosa and colleagues (2021), which revealed that 8 infants responded to a live music theatre performance with high levels of attention (62\%), moderate levels of positive affect (8\%) and low levels of negative affect (3\%). Thirty-one caregivers filled out an optional post-performance survey and reported that their infants were either neutral about the show (23\%), enjoyed it (42\%), or really enjoyed it (35\%). Most caregivers (80\%) reported that they also either enjoyed or really enjoyed the show.

Alongside high interest in the show overall, infants' attention was modulated by the performed song. Paralleling laboratory work, the playsong captured infant attention more than the lullaby (Cirelli et al., 2020; Rock et al., 1999). These attentional differences have been interpreted as reflective of intended song function - while playsongs are intended to engage alert infants, lullabies should generate more internalized responses (Rock et al., 1999). Along 
1 these line, it is important to note that infants do not need to attend visually to the source of

2 music to perceive the sound and experience emotional effects: though lullabies do not capture

3 visual attention, they reduce physiological arousal as intended (Cirelli et al., 2000). The patterns

4 of attention over time were remarkably consistent within song type and across performances,

5 regardless of caregiver engagement (see Supplemental Figure 3). In laboratory settings, when

6 caregivers are asked to sing the same song as either a lullaby or playsong, lullaby renditions

7 are slower, softer, and lower (Cirelli et al., 2020). Given the naturalistic context of this

8 performance, the lullaby and playsong, composed and arranged specifically with these

9 functional goals in mind, differed in a variety of ways beyond these basic acoustic properties.

10 This performance was also multimodal - infants listened to the singers and instrumentalists but

11 also watched the performers emote and move. While it is beyond the scope of the current study

12 to identify which features of the performance drove the differences in infant responses across

13 lullaby and playsong conditions, it is striking that infants' behavioral responses to song style

14 generalize to contexts beyond the caregiver-infant dyad.

15 At the same time, our results showed that the caregiver-infant unit was relevant even in

16 the group context. Infants' attention to and away from the performance was more coordinated

17 with their own caregiver than with another caregiver watching the same performance. The

18 coordination of visual attention between infants and caregivers facilitates information exchange,

19 language development, and social-emotional bonding (Feldman, 2007; Piazza et al., 2020; Salo

20 et al., 2018; Tomasello et al., 2005). Coordinated attention in this musical context suggests that

21 infants and caregivers were sensitive to each other's attention to the show and to one another,

22 above and beyond the features of the show itself. Even within the context of a moderately large

23 audience (which comprised $\sim 50$ other people total), this hallmark of caregiver-infant connection

24 was still evident. It should be noted that joint attention in the literature usually refers to shared

25 focus on a specific object, concept, or feature, which could not be confirmed with the video data

26 we were able to capture. Future research could use more detailed measures of visual attention, 
1 such as mobile eye-tracking, to explore whether caregivers and infants attend to the same

2 elements of the performance, in addition to their coordinated attention to the performance 3 generally.

We found no evidence to suggest that infant attention to the performance was influenced

5 by whether their caregivers were instructed to be passive or interactive audience members.

6 When they were instructed to provide an interactive experience, caregivers spent more time

7 moving rhythmically, and were more likely to look at their infants, perhaps engaging in bids for

8 joint attention. It is possible that caregivers also adjusted their behavior in ways that cannot be

9 accounted for in our video coding. For example, during the playsong, the singer used hand

10 motions and iconic gestures that caregivers might have mimicked, or caregivers may have

11 adjusted their use of affective touch, but these movements would not necessarily have been

12 rhythmic and therefore would not have been included in our coding. Similarly, because the

13 research assistants coded behaviors without sound, vocalizations were not captured.

These differences in caregiver behavior across engagement conditions influenced

15 infants' emotional responses differentially for each song type. For the playsong, the interactive condition encouraged more infant smiles and laughter than the passive condition. The playsong

17 used here specifically included moments where caregivers could perform specific actions and

18 included fun moments of suspense. Previous research suggests that, in non-musical contexts,

19 infant smiles and laughter are largely driven by cognitive appraisals of absurdity or surprise,

20 rather than by caregiver laughter (Mireault at al., 2018). Caregiver engagement in the playsong

21 condition may have enhanced infants' attention to surprising moments in the song (e.g., a

22 performer suddenly exclaiming "SPLAT?"). The playsong also included lyrics about tickling at

23 two timepoints ("tickle your nose, tickle your toes"). Although about $70 \%$ of all smiles happened

24 outside of these timepoints (which were omitted in our analyses of infant affect), tickling during

25 those moments may have generated smiles not only during the tickles, but also increased the

26 likelihood of smiles as the song progressed. Playsongs often include iconic gestures that may 
encourage caregivers to touch their infants, which likely have important impacts on infants'

2 engagement and affective responses to these songs. For the lullaby condition, infant affect was

3 not influenced by engagement condition.

Most infants (80\%) generated rhythmic movements at some point during the experiment.

5 Clapping, swaying, arm movements, leg swinging, head bobbing, and bouncing were among the

6 most common movements. Neither infant age nor song style influenced infant rhythmic

7 movements. The lack of association between age and rhythmic movements may be surprising,

8 given the vast differences in the motor capabilities of the average 6- and 18-month-old. This

9 null association is potentially attributable to large individual differences in age of onset for infant

10 musical movements (Kim \& Schachner, 2019). Perhaps like other motor abilities that come

11 online in infancy (e.g., walking), experience performing that motor skill (e.g., weeks since

12 walking onset) predict skill more than age (Rachwani et al., 2020). Consistent with that

13 explanation, we observed suggestive evidence that the duration of time infants moved

14 rhythmically was associated the Parent Initiation of Musical Behavior subscale of the

15 Music@Home Infant questionnaire (Politimou et al., 2018). The "Parent Initiation of Music-

16 Making" score probes the regularity with which caregivers integrate music-making into their

17 interactions with their infants. Infants' own experiences moving to music at home, therefore,

18 may be a salient factor for whether they move to music in an audience. On the group level,

19 infants spent more time moving rhythmically in the passive than the interactive caregiver

20 engagement conditions. This may reflect an early desire to move or be moved in musical

21 contexts. If infants desire movement in a musical context, then in the interactive condition this

22 desire is fulfilled by caregiver movements. In the passive condition, when caregivers remain still,

23 self-generated movements are required. Given the low rate of movement overall and the lack of

24 a non-musical control condition, this explanation is highly tentative and warrants further

25 investigation. 
The present sample represented fairly affluent families with high cultural and linguistic diversity. Emerging research suggests that community musical events for infants and caregivers

3 may be especially impactful for families with less social support, who may be more likely to

4 integrate music making into their daily routines after attending these events (Rodrigues et al.,

5 2010; Gudmundsdottir \& Gudmundsdottir, 2010; Fancourt \& Perkins, 2018; Lense et al., 2020).

6 Alongside this research is a growing recognition for the need to study musical experiences in

7 more ecologically valid contexts. Increasing global interest in infant-directed musical

8 performances provides an excellent avenue to explore the earliest roots of group-based musical

9 experiences, and the generalizability of the reported effects. While many infants participating in

10 this study had experienced music in a group setting in the past (e.g., infant group music

11 classes), it is unlikely that they were familiar with operatic styles of musical performances. It is

12 also possible that this style of music is most relevant and accessible for families within specific

13 social-cultural groups. However, this musical style highlights the voice and emotionality, which

14 are often important components of infant-directed musical performances.

In sum, the results of this naturalistic experiment suggest that musical experiences in a

16 live group setting are engaging, even early in life. Our results replicate and extend laboratory

17 work showing that song function (to soothe or to engage in play) has distinct effects on infant

18 behavior (Cirelli et al., 2020). Results show this experience was highly social - infants share this

19 experience with their caregiver and have different emotional and rhythmic responses depending

20 on caregiver engagement and song style. In short, infants are attentive audience members, and

21 their responses are shaped by elements of the performance itself, their caregivers' reactions,

22 and their own musical histories.

\section{References}

25 Ardizzi, M., Calbi, M., Tavaglione, S., Umiltà, M. A., \& Gallese, V. (2020). Audience

26 spontaneous entrainment during the collective enjoyment of live performances: 
Physiological and behavioral measurements. Scientific Reports, 10(1), 3813. https://doi.org/10.1038/s41598-020-60832-7

Bainbridge, C. M., Bertolo, M., Youngers, J., Atwood, S., Yurdum, L., Simson, J., Lopez, K., Xing, F., Martin, A., \& Mehr, S. A. (2020). Infants relax in response to unfamiliar foreign lullabies. Nature Human Behaviour. https://doi.org/10.1038/s41562-020-00963-z

Barbosa, M., Vences, M., Rodrigues, P. M., \& Rodrigues, H. (2021). Babies' engagement in music theater performances: A microanalytical study of the aesthetic experiences in early childhood. Psychology of Aesthetics, Creativity, and the Arts. https://doi.org/10.1037/aca0000379

Belfi, A. M., Samson, D. W., Crane, J., \& Schmidt, N. L. (2021). Aesthetic judgments of live and recorded music: Effects of congruence between musical artist and piece. Frontiers in Psychology, 12, 618025. https://doi.org/10.3389/fpsyg.2021.618025

Brooks, M.E., Kristensen, K., van Benthem, K.J., Magnusson, A., Berg, C.W., Nielsen, A., Skaug, H.J., Maechler, M., Bolker, B.M. (2017). glmmTMB balances speed and flexibility among packages for zero-inflated generalized linear mixed modeling. The $R$ Journal, 9(2), 378-400. https://journal.r-project.org/archive/2017/RJ-2017066/index.html.

Brown, S. C., \& Knox, D. (2017). Why go to pop concerts? The motivations behind live music attendance. Musicae Scientiae, 21(3), 233-249. https://doi.org/10.1177/1029864916650719

Cirelli, L. K., Jurewicz, Z. B., \& Trehub, S. E. (2020). Effects of maternal singing style on mother-infant arousal and behavior. Journal of Cognitive Neuroscience, 32(7), 12131220. https://doi.org/10.1162/jocn_a_01402

Cirelli, L. K., \& Trehub, S. E. (2020). Familiar songs reduce infant distress. Developmental Psychology, 56(5), 861. https://doi.org/ 10.1037/dev0000917 
1 Dotov, D., Bosnyak, D., \& Trainor, L. J. (2021). Collective music listening: Movement energy is enhanced by groove and visual social cues. Quarterly Journal of Experimental Psychology, 74(6), 1037-1053. https://doi.org/10.1177/1747021821991793

Drury, R. C., \& Fletcher-Watson, B. (2017). The infant audience: The impact and implications of child development research on performing arts practice for the very young. Journal of Early Childhood Research, 15(3), 292-304. https://doi.org/10.1177/1476718X15614041

Egermann, H., Sutherland, M. E., Grewe, O., Nagel, F., Kopiez, R., \& Altenmüller, E. (2011). Does music listening in a social context alter experience? A physiological and psychological perspective on emotion. Musicae Scientiae, 15(3), 307-323. https://doi.org/10.1177/1029864911399497

Fancourt, D., \& Perkins, R. (2018). Effect of singing interventions on symptoms of postnatal depression: three-arm randomised controlled trial. The British Journal of Psychiatry: The Journal of Mental Science, 212(2), 119-121. https://doi.org/10.1192/bjp.2017.29

Feldman, R. (2007). Parent-infant synchrony: Biological foundations and developmental outcomes. Current Directions in Psychological Science, 16(6), 340345. https://doi.org/10.1111/j.1467-8721.2007.00532.x

Fletcher-Watson, B., Fletcher-Watson, S., McNaughton, M. J., \& Birch, A. (2014). From cradle to stage: How early years performing arts experiences are tailored to the developmental capabilities of babies and toddlers. Youth Theatre Journal, 28(2), 130-146. https://doi.org/10.1080/08929092.2014.940075

Gudmundsdottir, H. R., \& Gudmundsdottir, D. G. (2010). Parent-infant music courses in Iceland: Perceived benefits and mental well-being of mothers. Music Education Research, 12(3), 299-309. https://doi.org/10.1080/14613808.2010.505644

Ilari, B. (2005). On musical parenting of young children: Musical beliefs and behaviors of mothers and infants. Early Child Development and Care, 175(7-8), 647-660. https://doi.org/10.1080/0300443042000302573 
1 Kim, M. \& Schachner, A. (2019, March). The origins of dance: Characterizing the development of infants' earliest dance behavior. Poster presented at the meeting of the Society for Research in Child Development, Baltimore, MD, USA.

4 Krause, A. E., Maurer, S., \& Davidson, J. W. (2020). Characteristics of self-reported favorite musical experiences. Music \& Science. https://doi.org/10.1177/2059204320941320

6 Lamont, A. (2011). University students' strong experiences of music: Pleasure, engagement, 249. https://doi.org/10.1177/1029864911403368

Lausberg, H. \& Sloetjes, H. (2009). Coding gestural behavior with the NEUROGES-ELAN system. Behavior Research Methods, 41, 841-849. https://doi.org/10.3758/BRM.41.3.841

Lense, M. D., Beck, S., Liu, C., Pfeiffer, R., Diaz, N., Lynch, M., Goodman, N., Summers, A. \& Fisher, M. H. (2020). Parents, peers, and musical play: Integrated parent-child music class program supports community participation and well-being for families of children with and without Autism Spectrum Disorder. Frontiers in Psychology, 11, 555717. https://doi.org/10.3389/fpsyg.2020.555717

Mendoza, J. K., \& Fausey, C. M. (in press). Everyday music in infancy. Developmental Science.

Piazza, E. A., Hasenfratz, L., Hasson, U., \& Lew-Williams, C. (2020). Infant and adult brains are coupled to the dynamics of natural communication. Psychological Science, 31(1), 6-17. https://doi.org/10.1177/0956787619878698

Politimou, N., Stewart, L., Müllensiefen, D., \& Franco, F. (2018). Music@Home: A novel instrument to assess the home musical environment in the early years. PLOS ONE, 13(4), e0193819. https://doi.org/10.1371/journal.pone.0193819

Rachwani, J., Hoch, J., \& Adolph, K. (2020). Action in development: plasticity, variability, and flexibility. In J. Lockman \& C. Tamis-LeMonda (Eds.), The Cambridge Handbook of 
Infant Development: Brain, Behavior, and Cultural Context (pp. 469-494). Cambridge University Press. https://doi.org/10.1017/9781108351959.017

R Core Team. (2020). R: A language and environment for statistical computing. R Foundation for Statistical Computing, Vienna, Austria. https://www.R-project.org/

Rock, A. M. L., Trainor, L. J., \& Addison, T. L. (1999). Distinctive messages in infant-directed lullabies and play songs. Developmental Psychology, 35(2), 527-534. https://doi.org/10.1037/0012-1649.35.2.527

Salo, V. C., Rowe, M. L., \& Reeb-Sutherland, B. (2018). Exploring infant gesture and joint attention as related constructs and as predictors of later language. Infancy, 23(3), 432452. https://doid.org/10.1111/infa.12229

Savage, P. E., Loui, P., Tarr, B., Schachner, A., Glowacki, L., Mithen, S., \& Fitch, W. T. (2020). Music as a coevolved system for social bonding. Behavioral and Brain Sciences, 1-36. https://doi.org/10.1017/S0140525X20000333

Shoda, H., Adachi, M., \& Umeda, T. (2016). How live performance moves the human heart. PLOS ONE, 11(4), e0154322. https://doi.org/10.1371/journal.pone.0154322

Steegen, S., Tuerlinckx, F., Gelman, A., \& Vanpaemel, W. (2016). Increasing transparency through a multiverse analysis. Perspectives on Psychological Science, 11(5), 702-712. https://doi.org/10.1177/1745691616658637

Swarbrick, D., Bosnyak, D., Livingstone, S. R., Bansal, J., Marsh-Rollo, S., Woolhouse, M. H., \& Trainor, L. J. (2019). How live music moves us: Head movement differences in audiences to live versus recorded music. Frontiers in Psychology, 9, 2682. https://doi.org/10.3389/fpsyg.2018.02682

Tomasello, M. Carpenter, M., Call, J., Behne, T., \& Moll, Henrike. (2005). Understanding and sharing intentions: The origins of cultural cognition. Behavioral and Brain Sciences, 28, 675-735. https://doi.org/0.1017/S0140525X05000129 
1 Trehub, S. E., \& Gudmundsdottir, H. R. (2015). Mothers as singing mentors for infants. The Oxford Handbook of Singing, 454-470. https://doi.org/10.1093/oxfordhb/9780199660773.013.25

4 Upham, F., \& McAdams, S. (2018). Activity analysis and coordination in continuous responses to music. Music Perception, 35(3), 253-294. https://doi.org/10.1525/mp.2018.35.3.253 
Figure Legends

2 Figure 1. The performance took place in an auxiliary room of the city's performing arts center.

3 The performers (singers, pianist, and auxiliary percussionist) were positioned at the front of the

4 room. The audience was in three rows with an aisle in the middle for easy access. Cameras on

5 tripods were positioned around the audience to capture the infants' behaviors.

7 Figure 2. Kernel density plots (smoothed histograms) depicting the proportion of time infants

8 attended to the show during each song and engagement condition.

$9 \quad{ }^{*} p<.05,{ }^{* *} p<.01,{ }^{* *} p<.001$

10

11 Figure 3. Kernel density plots (smoothed histograms) depicting the proportion of time infants'

12 attention was consistent with either their own caregiver or a random caregiver (both looking at

13 the show, at each other, or at neither).

$14 \quad{ }^{*} p<.05,{ }^{* *} p<.01,{ }^{* * *} p<.001$

15

16 Figure 4. (A) Depicts the proportion of time infants showed positive facial expressions (smiling,

17 laughing) during each trial. Error bars represent 95\% within-subjects confidence intervals. (B)

18 Depicts the number of infants who expressed negative affect (frowning, crying) during the trial.

$19{ }^{*} p<.05,{ }^{* *} p<.01,{ }^{* * *} p<.001$

20 